\title{
Isolation of Chlorpyrifos Degrading Bacteria from Garden Soil
}

\section{Rajesh $\mathbf{M}^{*}$, Archana B and Samundeeswari M}

PG \& Research Department of Zoology, The American college, India

*Corresponding author: Rajesh M, PG \& Research Department of Zoology, The American college, Madurai, Tamil Nadu, India, Tel: 9443394233; Email: rajesh@americancollege.edu.in

\section{Research article}

Volume 2 Issue 3

Received Date: May 07, 2019

Published Date: June 07, 2019

DOI: $10.23880 /$ izab- 16000155

\section{Abstract}

Chlorpyrifos is an organophosphorus insecticide broadly used for pest control in the urban and domestic area. It plays an important role in agriculture. Because of its persistent usage, it remains in the natural environment for a long period and hard to degrade its intermediate compounds. Microbial degradation is the best way to confiscate its toxicity. The present study reported that the degradation of chlorpyrifos by soil Pseudomonas aeruginosa Soil sample was collected from the garden. After enriching the soil sample with chlorpyrifos pesticide, the bacterial strain was isolated. The isolated bacterium was identified as Pseudomonas aeruginosa and used for chlorpyrifos degradation studies. The efficient production of biosurfactant by Pseudomonas aeruginosa bacterium was tested with the experiments. The presence of biosurfactant property increases the rate of degradation of chlorpyrifos by Pseudomonas aeruginosa. The efficiency of the bacterial strain on the degradation of different concentration of the chlorpyrifos was studied using 1, 5 and 9 ppm of the chlorpyrifos for 15 days. HPLC analysis showed that there was a reduction in peak area of the different concentrations of pesticide samples (1,5 and $9 \mathrm{ppm}$ ) inoculated with the isolated Pseudomonas aeruginosa with that of control pesticide sample without inoculum on different days (5,10 and 15th day). The bacteria effectively degraded the chlorpyrifos in all the concentration when the duration of exposure is increased. From these above studies, it has been concluded that chlorpyrifos can be biodegradable by Pseudomonas aeruginosa bacteria which can utilize carbon, nitrogen and phosphorous sources from chlorpyrifos pesticide.

Keywords: Chlorpyrifos; Pseudomonas aeruginosa; Biosurfactant; Biodegradation; HPLC

\section{Introduction}

Pesticides are chemical agents which kill pests especially insects. Pesticides have numerous beneficial effects which include crop protection, preservation of food materials and to control vector-borne diseases. Chlorpyrifos is widely used for pest control in agriculture, gardening and soil treatment [1]. Among pesticides, Organophosphate pesticides are a highly toxic group of agricultural chemicals which widely used to increase plant protection and production. Abundant usage of pesticide resulted in an accumulation of pesticide residues in crops, soils, and biosphere creating an environmental stress [2].

Chlorpyrifos, (0, 0-diethyl 0-(3,5,6-trichloro-2-pyridyl) phosphorothioate) is one of the most commonly used broad-spectrum Organophosphates, and its phosphorus is 
linked to a sulfur with a double bond $(\mathrm{P}=\mathrm{S})$. It is used globally to control a variety of chewing and sucking insect pests and mites on the economically important crops including rice, bananas, vegetables, and cotton etc. Applicability of Chlorpyrifos also causes serious damage to non-target organisms [3]. A consequence of the persistent usage of chlorpyrifos causes contamination in an aquatic and terrestrial environment [4]. In previous studies, chlorpyrifos may affect all the physiological systems due to its high mammalian toxicity $[5,6]$. Compounds of this organophosphate pesticide are hydrolyzed spontaneously and cause major neurotoxicity in mammals [7].

The half-life of chlorpyrifos is 60-120 days in soil and its rate of degradation is 50 to 570 days based on the nature of the soil [8]. During degradation the chlorpyrifos converted into 3, 5, 6-trichloro-2-pyridinol (TCP), an intermediate compound accumulate in the natural environment, which is recalcitrant to microbial degradation [9]. TCP has an antimicrobial property that prevents the proliferation of chlorpyrifos degrading microbial population [10].

Detoxification of chlorpyrifos intermediates using microorganisms is comparatively a best alternative approach of pesticide degradation because other methods of removing chlorpyrifos residues are impractical or costly or are themselves hazardous to the environment [11].

Biosurfactants are amphipathic in nature. It has a characteristic feature of hydrophilic-lipophilic balance, which specifies the portion of hydrophilic and hydrophobic constituents in surface-active substances. Because of their amphiphilic structure, biosurfactants increase the bioavailability of hydrophobic contaminants by changing the properties of the bacterial cell surface [12].

In the present study, Pseudomonas aeruginosa was isolated from pesticide-treated garden soil invitro biodegradation of Chlorpyrifos was performed. Degradation capacity of isolated Pseudomonas aeruginosa was identified by HPLC analysis and its biosurfactant ability was also assessed by blue agar plate method and penetration assay.

\section{Materials and Methods}

\section{Chemical}

The organophosphate Chlorpyrifos (Emulsifiable Concentrate $(E C)=20 \%$ ) was used in the present study.
Commercial-grade insecticide chlorpyrifos (20\% EC) was obtained from the pesticide shop, Madurai, Tamil Nadu, India. It was used throughout the experimental studies, because it may more closely resemble the active compound that microorganisms are likely to be exposed to in the soil environment and HPLC grade methanol was used.

\section{Media}

Nutrient broth, nutrient agar medium, Pseudomonas isolation agar medium and minimal salts broth were used for chlorpyrifos degradation studies.

\section{Collection of Soil Sample}

The soil samples were collected from the garden at The American College campus. The soil was collected randomly from $0-25 \mathrm{~cm}$ depth from four corners 4-6 meters part and from a center of the field. The samples were pooled together, brought to the laboratory in polyethylene bags. The collected soil samples were ground, passed through $2 \mathrm{~mm}$ sieve and stored in a refrigerator at $5-6^{\circ} \mathrm{C}[13]$.

\section{Enrichment of the Soil Samples}

The aim of this step was to adapt the soil microflora to insecticide, chlorpyrifos. To obtain this effect, collected soil samples $(5 \mathrm{~g})$ were mixed with $100 \mathrm{ml}$ chlorpyrifos at different concentrations such as $1 \mathrm{ppm}, 5 \mathrm{ppm}$, and $9 \mathrm{ppm}$. Chlorpyrifos was introduced in a form of solution. After mixing, the soil suspension was incubated in the dark at 300C. After 10-15 days of incubation, the aliquot of chlorpyrifos treated soil suspension was taken for isolation of bacteria [14].

\section{Isolation and Identification of Bacteria from Soil Sample}

$1 \mathrm{~g}$ of pesticide-treated soil sample was weighed and kept in a test tube. $10 \mathrm{ml}$ of sterile distilled water was added to the soil sample, mixed well and it was serially diluted nine times to arrive at 10-9 concentration, using a sterile pipette. Then the diluted sample was used in spread plate technique.

The plates were then incubated for 24 hours at $370^{\circ} \mathrm{C}$ to allow the formation of colonies. One colony was selected from the plates and pure cultures were developed through streak plate method. Then the selected strain is sub cultured in the nutrient broth. The isolated strain was morphologically identified by gram staining. The slide was air dried and cell morphology was checked 


\section{International Journal of Zoology and Animal Biology}

under the microscope. The isolated strain was identified up to genus level by biochemical tests.

\section{Screening of Biosurfactant Producing Organisms}

The isolated strain was tested for their biosurfactant production by Penetration assay and Blue agar plate method $[15,16]$.

\section{Results}

\section{Isolation and Identification of Chlorpyrifos Degrading Bacteria}

A single microorganism was isolated from the chlorpyrifos-exposed soil samples by spread plate method. The isolate was found to possess the ability to degrade the organophosphate pesticide. The strain was a rod-shaped, gram-negative, a motile bacterium. Based on the morphological and biochemical characteristics the isolated strain was identified as Pseudomonas species (Table 1).

\begin{tabular}{|c|c|c|}
\hline S. No & Biochemical test & Results \\
\hline 1 & Gram stain & Gram negative, Rod, Motile \\
\hline 2 & Xylose & Positive \\
\hline 3 & Maltose & Negative \\
\hline 4 & Arabinose & positive \\
\hline 5 & Malonate & Positive \\
\hline 6 & Urea & Positive \\
\hline 7 & Trehalose & Negative \\
\hline 8 & Fructose & Negative \\
\hline 9 & Lysine & Negative \\
\hline 10 & Arginine & Positive \\
\hline 11 & Pyruvate & Positive \\
\hline 12 & Ornithine & Negative \\
\hline 13 & Sucrose & Negative \\
\hline 14 & Inositol & Negative \\
\hline 15 & Aesculin & Negative \\
\hline 16 & Citrate & Positive \\
\hline 17 & Sorbitol & Negative \\
\hline 18 & Mannitol & Negative \\
\hline 19 & Arabitol & Positive \\
\hline 20 & Raffinose & Negative \\
\hline 21 & Cellobiose & Negative \\
\hline 22 & Agmatine & Negative \\
\hline 23 & Catalase & Positive \\
\hline
\end{tabular}

Table 1: Biochemical tests for Pseudomonas species.

Biochemical analysis: The isolated strain showed the positive results of the following biochemical tests such as
Xylose, Arabinose, Malonate, Urea, Arginine, Pyruvate, Citrate, Arabitol and Catalase (Figure1).

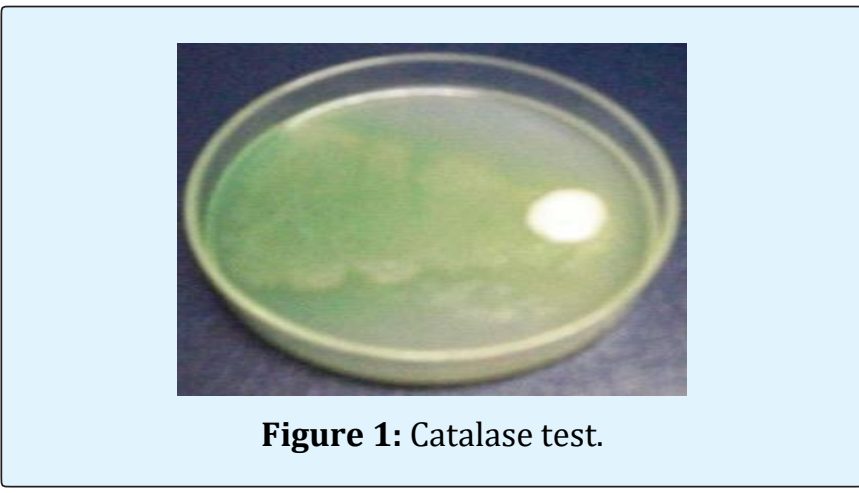

Selective media isolation: The isolated strain was grown on the Pseudomonas isolation agar medium. This result confirmed that the isolated bacterium was Pseudomonas aeruginosa (Figure 2). In addition to that further characterization was done by biochemical analysis in Bose clinical laboratory, Simmakal, Madurai, Tamil Nadu, India. It strengthened the result as Pseudomonas aeruginosa.

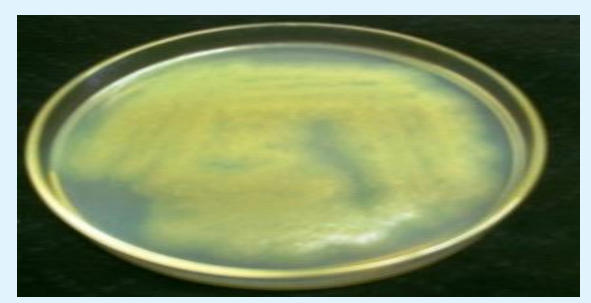

Figure: 2: Growth of the Pseudomonas aeruginosa.

\section{Screening of Biosurfactant Producing Pseudomonas Aeruginosa}

In the present study, the bacterial strain Pseudomonas aeruginosa was isolated from pesticide-contaminated soil samples by plate and dilution techniques. They were further screened for biosurfactant property by penetration assay and blue agar plate method.

Penetration Assay: This assay contained oil and silica gel paste in a 96 well microplate. The colored supernatant is placed on the surface of the paste. The upper phase changed from red to cloudy white within 15 minutes. The remaining portion also became red in color because the dye dissolved in it. This indicated the bacterial strain Pseudomonas aeruginosa had the capacity to produce biosurfactant (Figure 3). 


\section{International Journal of Zoology and Animal Biology}

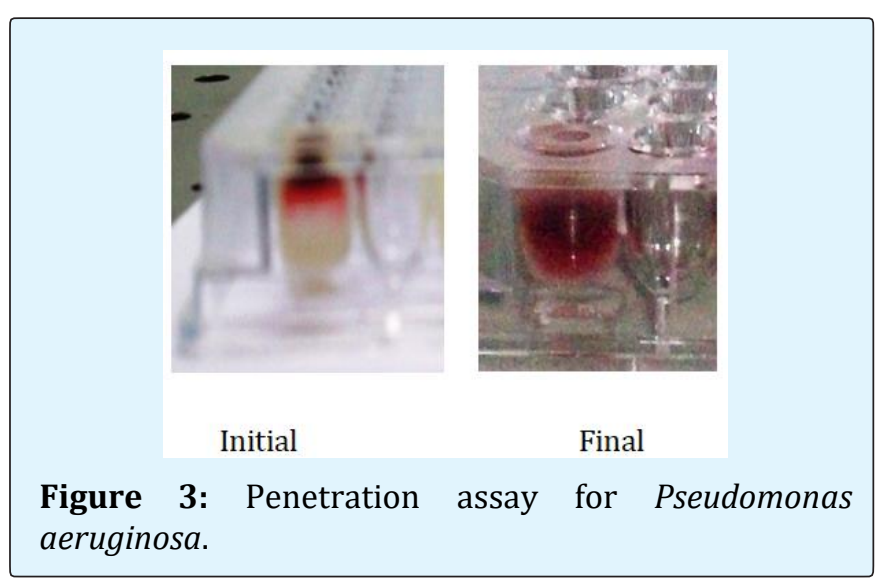

Blue agar plate method : In the methylene blue agar plate supplemented with CTAB, fifty microlitres of cellfree supernatant was loaded into the wells. The plate was incubated at $370^{\circ} \mathrm{C}$. After 48 hours a dark blue halo zone was formed and it confirmed the presence of anionic biosurfactant (Figure 4).

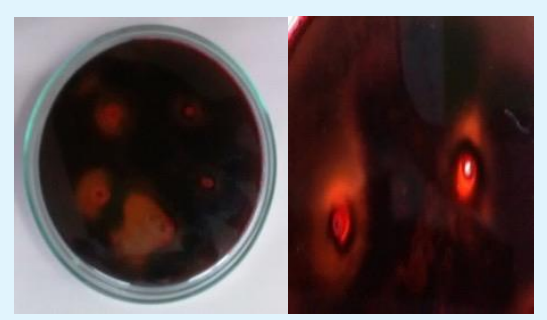

Figure 4: Blue agar plate method for Pseudomonas aeruginosa- Dark blue halo zone formation.

\section{Discussion}

Results of the present study were based on the biodegradation potential. The organism isolated from the garden soil was identified as Pseudomonas aeruginosa based on the morphological and biochemical characterizations. The rate of biodegradation is increased by the Pseudomonas aeruginosa which has the capacity to produce biosurfactants.

For this study, chlorpyrifos resistant bacterium was isolated by enriching soil sample which is contaminated with the organophosphate chlorpyrifos. The isolated microorganism is allowed to grow on the Pseudomonas isolation agar medium. This medium is very useful for isolating Pseudomonas species. Pseudomonas Isolation Agar includes Irgasan which has a potent antimicrobial activity but not active against Pseudomonas species and it selectively inhibits Gram-positive and Gram-negative bacteria other than Pseudomonas species. This medium is discerning and prepared to enhance the formation of blue or blue-green pyocyanin pigment because this confirms the presence of Pseudomonas aeruginosa. The production of pyocyanin pigment was promoted by the presence of Magnesium Chloride and Potassium Sulfate in the medium [17].

The rate of degradation of methyl parathion which is an organophosphorus pesticide was found out by using physical techniques such as UV-visible spectroscopy, FTIR spectroscopy, and HPLC. The extent of biodegradation was evident from the reduction of the peak and it is also observed from the decrease of area of the peak [18].

In the present study, the peak area of the experimental sample reduced when compared to the control. Pesticide concentration is one of the major factors that determine the fate of biodegradation. Very high concentration leads to the failure of biodegradation as microbes are not resistant against that. On the other hand, very low pesticide concentration shows a strong affinity with soil particles thus become non-available to microbes [19]. The rate of degradation is based on the concentration of Chlorpyrifos. Hence, the area measurement is a more reliable measure of the concentration of chlorpyrifos. The media contained a $1 \mathrm{ppm}$ concentration of Chlorpyrifos had higher peak area when compared to the 5 and 9 ppm concentration of chlorpyrifos. In each concentration, the peak area decreased gradually from $5^{\text {th }}$ day to $15^{\text {th }}$ day. This indicated that the rate of chlorpyrifos degraded gradually by Pseudomonas aeruginosa. Chlorpyrifos was used as a carbon source, phosphorous and energy source by the microorganisms. There is no additional energy source added in this present study to assure the degrading capability of bacterial isolates [20].

Another reason of efficient degradation is the Pseudomonas aeruginosa has the capacity to produce biosurfactants. Pseudomonas species, Bacillus species, and Acinetobacter species have potential to produce biosurfactant for acceleration of biodegradation of pesticides. It increased the biodegradation efficiency of chlorpyrifos by improving the bioavailability of the xenobiotic compound by developing critical micellar concentration. The complete detoxification of the pesticide occurs with those microbes that also degrade its metabolites and avoid its accumulation in the environment. The biosurfactant production, mineralization ability, immobilization of cells, nutrient supplementation, etc. increased the activity of microbial strains in chlorpyrifos degradation [21]. 


\section{International Journal of Zoology and Animal Biology}

Pseudomonas aeruginosa showed the highest production of biosurfactant in both cell-free culture and pellets [22]. Similarly, in the present study, Pseudomonas aeruginosa produce biosurfactant. The production of biosurfactant by Pseudomonas aeruginosa was determined by using two qualitative methods such as penetration assay and blue agar plate method. The penetration assay relies on the contacting of two insoluble phases which lead to a color change. In this assay, the color changed within 15 minutes from clear red to cloudy white. The described effect relies on the phenomenon that silica gel is entering the hydrophilic phase from the hydrophobic paste much more quickly because of the presence of biosurfactants producing capacity. In penetration assay, the presence of biosurfactant was confirmed by the experiment done in Nocardiopsis $s p$. with remarkable bio-surfactant and biodegradation capacity [23].

In the blue agar plate method dark blue halo zone was formed after 48 hours of incubation. This is because of the production of rhamnolipid by Pseudomonas aeruginosa. The dark blue halo zone in the methylene blue agar plate supplemented with CTAB confirmed the presence of anionic biosurfactant. The assay was developed based on the property that the concentration of anionic surfactants in aqueous solutions can be determined by the formation of insoluble ion pairs with various cationic substances. The insoluble ion pair precipitates are formed in the agar plate containing methylene blue exhibited dark blue color. The diameter of the dark blue region has been shown to be semi-quantitatively proportional to the concentration of the rhamnolipid biosurfactants [24].

The biosurfactant produced by microorganism was found to be specific to organophosphates and it also plays a major role in enhancing the degradation processes of organophosphates $[25,26]$. In this study, the Pseudomonas aeruginosa degrades chlorpyrifos gradually. The rate of degradation is increased with the help of biosurfactant properties.

\section{Conclusion}

The Pseudomonas aeruginosa isolated from the garden soil effectively degraded the chlorpyrifos in all the concentration when the duration of exposure is increased. From the studies, it has been concluded that chlorpyrifos can be biodegradable and Pseudomonas aeruginosa utilized carbon, nitrogen and phosphorous from chlorpyrifos pesticide for its growth. This strain can be used for bioremediation of chlorpyrifos contaminated sites.

\section{Acknowledgement}

I am thankful to The American College for providing me with the facilities and allowing me to carry out my project work. I take this opportunity to express my sincere gratitude to my guide Prof. M. Rajesh, for inspiring, guidance, affectionate encouragement, care and support throughout my project. Though words are not enough, it is my duty and heartiest wish to greatly acknowledge and thank Dr. Ted Adams for providing me educational support.

\section{References}

1. Crespo Corral E, Santos Delgado MJ, Polo Diez LM, Soria AC (2008) Determination of carbamate, phenylurea and phenoxy acid herbicide residues by gas chromatography after potassium tertbutoxide/dimethyl sulphoxide/ethyl iodide derivatization reaction. J Chromatogr A 1209(1-2): 22-28.

2. Qiao CL, Yan YC, Shang HY, Zhou XT, Zhang Y (2003) Biodegradation of pesticides by immobilized recombinant Escherichia coli. Bull Environ Contam Toxicol 71(2): 370-374.

3. Thengodkar RR, Sivakami S (2010) Degradation of chlorpyrifos by an alkaline phosphatase from the cyanobacterium Spirulina platensis. Biodegradation 21(4): 637-644.

4. Ruan QL, Ju JJ, Li YH, Li XB, Liu R, et al. (2012) Chlorpyrifos exposure reduces reproductive capacity owing to a damaging effect on gametogenesis in the nematode Caenorhabditis elegans. J Appl Toxicol, 32(7): 527-535.

5. Oliver GR, Bolles HG, Shurdut BA (2000) Chlorpyrifos: Probabilistic assessment of exposure and risk. Neurotoxicology 21(1-2): 203-208.

6. Tuzmen N, Candan N, Kaya E, Demiryas N (2008) Biochemical effects of chlorpyrifos and deltamethrin on altered antioxidative Defence mechanism and lipid peroxidation in rat liver. Cell Biochem Funct 26(1): 119-124.

7. Sogorb MA, Vilanova E (2002) Enzymes involved in the detoxification of organophosphorus, carbamate and pyrethroid insecticides through hydrolysis. Toxicology Letters 128(1-3): 215-228. 


\section{International Journal of Zoology and Animal Biology}

8. Singh BK, Walker A, Morgan JAW, Wright DJ (2003) Effects of soil $\mathrm{pH}$ on the biodegradation of chlorpyriphos and isolation of a chlorpyrifosdegrading bacterium. Appl and Environ Microbiol 69(9): 5198-5206.

9. Xu G, Zheng W, Li Y, Wang S, Zhang J, et al. (2008) Biodegradation of chlorpyrifos and 3,5,6 trichloro-2pyridinol by a newly isolated Paracoccus sp. strain TRP. International Journal of Biodeterioration \& Biodegradation 62(1): 51-56.

10. Li JQ, Liu J, Shen WJ, Zhao XL, Hou Y, et al. (2010) Isolation and characterization of 3,5,6-trichloro-2pyridinol-degrading Ralstonia sp. strain T6. Bioresour Technol 101(19): 7479-7483.

11. Horne I, Sutherland TD, Harcourt RL, Russell RJ, Oakeshott JG (2002) Identification of an opd (organophosphate degradation) gene in an Agrobacterium isolate. Appl Environ Microbiol, 68(7): 3371-3376.

12. Pacwa Plociniczak M, Plaza GA, Piotrowska Seget Z, Cameotra SS (2011) Environmental applications of biosurfactants: Recent advances. Int J Mol Sci 12(1): 633-654.

13. Gilani STS, Ageen M, Shah H, Raza S (2010) Chlorpyrifos Degradation in Soil and its Effect on Soil Microorganisms. The Journal of Animal \& Plant Sciences 20(2): 99-102.

14. Mahiudddin M, Fakhruddin ANM, Abdullah Al Mahin, Chowdhury MAZ, Rahman MA, et al. (2014) Degradation of the Organophosphorous Insecticide Diazinon by Soil Bacterial Isolate. The International Journal of Biotechnology 3(1): 12-23.

15. Walter V, Syldatk C, Hausmann R (2010) Screening Concepts for the Isolation of Biosurfactant Producing Microorganisms. Advanc Experiment Med Biol 672(113).

16. Saravanan V, Vijayakumar S (2012) Isolation and Screening of biosurfactant producing microorganisms from oil contamination soil. J Acad Indus Res 1(5): 264-268.

17. Collee JG, Fraser AG, Marmion BP, Simmons A (1996) Practical Medical Microbiology. In: Mackie, McCartney, (Eds.), Churchill Livingstone.
18. Ramachandran T, Violet Dhayabaran, Thirupathy, Jasmine R (2014) FTIR AND HPLC Analysis of Bacterial Degradation of Methyl Parathion. J Pharmacy and Pharmacertical Sciences 3(11): 929939.

19. Farhan M, Khan AU, Wahid A, Ahmad M, Ahamad F (2012) Biodegradation of Chlorpyrifos Using Indigenous Pseudomonas $s p$. Isolated from Industrial Drain, Pakistan. Journal of Nutrition 11(12): 11831189.

20. Pinjari AB, Novikov B, Rezenom YH, Russel DH, Wales $M E$, et al. (2012) Mineralization of acephate, a recalcitrant organophosphate insecticide is initiated by a Pseudomonad in environmental samples. PLOS One 7(4): e31963.

21. Dhanya MS (2014) Advances in microbial biodegradation of Chlorpyrifos. J Environ Res Develop 9(1): 232-240.

22. Sneha KS, Padmapriya, Rajeswari T (2012) Isolation and Screening of Biosurfactants Produced by Pseudomonas aeruginosa from Oil Spilled Soils. Int J Pharmaceut Biological Arch 3(2): 321-325.

23. Singh MJ, Sedhuraman P (2015) Report Biosurfactant, polythene, plastic, and diesel biodegradation activity of endophytic Nocardiopsis sp. mrinalini9 isolated from Hibiscus rosasinensis leaves. Bioresources and Bioprocessing 2(2): 1-7.

24. Siegmund I, Wagner F (1991) New method for detecting rhamnolipids excreted by Pseudomonas species during growth on mineral agar. Biotechnology Techniques 5(4): 265-268.

25. Sukirtha TH, Usharani MV (2013) Production and Qualitative Analysis of Biosurfactant and Biodegradation of the Organophosphate by Nocardia mediterranie. J Bioremed Biodeg 4(6): 1-8.

26. Li X, He J, Li S (2007) Isolation of a chlorpyrifosdegrading bacterium, Sphingomonas sp. Strain Dsp-2 and cloning of the mpd gene. Res Microbiol 158(2): 143-149. 